\title{
Smoking Cessation by Changing the Way of Smoking: Case Report
}

Pedro J Tarraga Lopez ${ }^{1 *}$, Loreto Tarraga-Marcos², Ibrahim Sadek ${ }^{3}$, Fatima Madrona-Marcos ${ }^{3}$, Alicia Vivo ${ }^{3}$ and Carmen Celada $^{4}$

${ }^{1}$ Department of Family Practice, Servicio de Salud de Castilla- La Mancha, Health Center number 5, Albacete, Albacete 02005, Spain

${ }^{2}$ Clinic Hospital Zaragoza, Servicio Aragones de Salud, Spain

${ }^{3}$ Department of Medicine, Servicio de Salud de Castilla-La Mancha, Albacete, Spain

${ }^{4}$ Department of Medicine, Servicio Murciano de Salud, Spain

\section{Abstract}

It is the case of a smoker woman over 45 years old who starts changing cigarettes to cigarettes without combustion with a significant improvement in the quality of life and at 9 months quit smoking completely.

Keywords: Smoker; Change smoking; Cigarettes without combustion; Harm reduction by tobacco

\section{Case Report}

Our patient is a 62 year-old woman, married with two children, working as a community pharmacist. As for the medical history she had no known medical allergies, hypertensive in treatment, no Diabetes Mellitus (DM), no Dyslipidemia, no heart or gastrointestinal diseases. She suffers from bronchopathy due to her smoking habit during the last 46 years, fulfilling the criteria of chronic bronchitis and bronchial hyperresponsiveness. Also she suffers from snoring without apneic breathing. Moderate-severe dental problems most probably due to tobacco smoking, requiring few dental implants. Incipient osteoporosis.

At the initial visit the examination was quite unremarkable.

During the physical examination our subject appeared alert, oriented and cooperative with an overall normal appearance and temperature. No signs of dyspnea at rest. Both cardiac and pulmonary auscultations were normal.

\section{Smoking History}

She was a regular smoker of 20 to 30 blond cigarettes daily for 46 years. Her husband also smokes. They have two children whom none of them smokes.

She had attempted to quit smoking in three occasions, two of them without any treatment and one with Varenicline, the last was the longest period of abstinence of tobacco she had (around 6 months). She commented that even during her 2 pregnancies she has not been able to stop smoking completely.

Her score in Fagerstrom Test for Nicotine Dependence was 6, 12 in Richmond with positive Reinforcement test.

In January 2018 after a dental intervention and due the anxiety that does not allow her to quit smoking, she changed her way of smoking into Heat-Not-Burn cigarettes.

She continued like this for 6 months during which she noticed improvement of her oropharyngeal halitosis and gastric dyspepsia. In June 2018, she consulted us to help her quit smoking, treating her anxiety with Bupropion $150 \mathrm{mg} / 24 \mathrm{~h}$. She has been smoking for two months and half, being relatively stable.

\section{Discussion}

A new strategy focuses on motivating smokers who cannot quit consuming nicotine in a low-risk way and reduce exposure to smoke toxins. A controversial measure. Smoking today is one of the biggest public health problems, being the first avoidable cause of disease, disability and premature death in the world. Although the prevalence of smoking has decreased in many developed countries, more than 1 billion people continue to smoke in the world nowadays $[1,2]$.

Many smokers want and try to quit, however, the long-term dropout rates are still very low. The public policies on tobacco control recommended by the World Health Organization are leading to an average annual decrease between $0.5 \%$ and $1 \%$ in the prevalence of smoking in developed countries, although the related mortality remains high.

Given the burden of diseases related to smoking, new strategies are needed to reduce their associated damage. Although it is important to continue motivating and helping people to stop smoking, it seems reasonable to look for other ways to reduce the harm of smoking as a complementary strategy to improve the health of smokers $[3,4]$. Harm reduction strategies focus on motivating smokers to consume nicotine in a low-risk way and reduce exposure to tobacco smoke toxins considerably, since nicotine, although not harmless by itself, does not produce chronic obstructive pulmonary disease, cardiovascular diseases or cancer. The implementation of these policies in smoking is a controversial issue: one of the main concerns is that this measure may be an obstacle to smoking cessation and may become an attraction to get the habit started, or the risk of ex-smokers suffers a relapse believing that the risks are acceptable. In our patient we have been able to verify that it has not been this way and although it was about 5 months with Heat-Not-Burn cigarettes, she is currently in the period of abandonment of the tobacco. There are theories that advocate the implementation of policies to reduce the harmful effects of tobacco as a complementary measure to other strategies for tobacco control [5].

In this regard, perhaps the best known is that expressed by the Royal College of Physicians of the United Kingdom, which, in the interest of public health, recommends promoting as widely as possible the use of new devices with nicotine as an alternative to the consumption of conventional cigarettes, considering that: 1) they do not constitute a form of habit initiation, since their use is limited almost exclusively to previous smokers; 2) there is no evidence that they represent a form of smoking initiation; 3) They help many smokers to try to quit; 4) They represent a health risk

*Corresponding author: Pedro J Tarraga Lopez, PH MD, Department of Family Practice, Servicio de Salud de Castilla-La Mancha, Health Center number 5, Albacete, Albacete 02005, Spain, Tel: 34609080627; Fax: 3467225533; Email: pjtarraga@sescam.jccm.es

Received: October 08, 2018; Accepted: October 31, 2018; Published: November 07,2018

Citation: Lopez PJT, Tarraga-Marcos L, Sadek I, Madrona-Marcos F, Vivo A, et al. (2018) Smoking Cessation by Changing the Way of Smoking: Case Report. Prim Health Care 8: 312. doi: 10.4172/2167-1079.1000312

Copyright: (c) 2018 Lopez PJT, et al. This is an open-access article distributed under the terms of the Creative Commons Attribution License, which permits unrestricted use, distribution, and reproduction in any medium, provided the original author and source are credited. 
substantially lower than that of tobacco; and 5) it is considered that, with adequate regulation, they have great potential to contribute favourably to the prevention of smoking and its associated diseases [6].

There are other examples, such as the FDA's new tobacco control framework or the evaluation carried out by the Norwegian Institute of Public Health to consider harm reduction as a strategic element in the control of smoking. Thus, the appearance of electronic nicotine release systems through heating has generated great expectations about its usefulness as a means to reduce the risks associated with smoking [7].

Most professionals have no doubts that tobacco products with reduced potential risk could help some people to achieve abandonment or reduce the health risks derived from smoking tobacco, as is the case of our patient who despite consuming this type of tobacco has seen the time to start the process of quitting smoking after so many years.

The available evidence supports that the new devices have the potential to achieve important public health benefits by reducing exposure to the toxic components of tobacco smoke and minimizing the damage caused by smoking. In clinical practice, physicians will increasingly consider the convenience (and the ethical dilemma) of recommending their use or providing information based on the available evidence that allows an informed decision-to those patients who cannot quit the habit or wish to reduce the health risks caused by tobacco $[8,9]$.

Our patient, for the moment, is in this favourable situation and we are convinced that after passing through the tobacco alternatives, she will achieve the final objective of tobacco cessation.

\section{References}

1. CAPLA (2018) About Tobacco.

2. Otero M, Ayesta FJ (2004) El tabaquismo como trastorno adictivo. Trastornos Adictivos 6: 78-88.

3. Silagy C, Lancaster T, Stead L, Mant D, Fowler G (2001) Nicotine replacement therapy for smoking cessation. Cochrane Database Syst Rev: CD000146.

4. Hughes JR, Stead LF, Lancaster T (2002) Antidepressants for smoking cessation. Cochrane Database Syst Rev 8: CD000031.

5. Mayoral RG, González FJC, Tornero Al, Cano AM, Agustín F, et al. (2016) Factores asociados al éxito de los tratamientos del tabaquismo. JONNPR 1: 131-137.

6. Clearing the smoke: Assessing the science base for tobacco harm reduction (2001) The National Academies Press.

7. JP Schalleer (2016) Evaluation of the tobacco heating system 2.2. Part 2: Chemical composition, genotoxicity, cytotoxicity, and physical properties of the aerosol. Regul Toxicol Pharmacol 81: S27eS47.

8. Lüdicke F, Picavet P, Baker G, Haziza C, Poux V, et al. (2018) Effects of switching to the tobacco heating system 2.2 menthol, smoking abstinence, or continued cigarette smoking on biomarkers of exposure: A randomized, controlled, openlabel, multicenter study in sequential confinement and ambulatory settings (Part 1). Nicotine Tob Res 20: 161-172.

9. Luedicke F, Picavet P, Baker G, Haziza C, Poux V, et al. (2018) Effects of switching to the menthol tobacco heating system 2.2, smoking abstinence, or continued cigarette smoking on clinically relevant risk markers: A randomized, controlled, open-label, multicenter study in sequential confinement and ambulatory settings (Part 2). Nicotine Tob Res 20: 173-182 